Check for updates

Cite this: RSC Adv., 2018, 8, 4946

Received 16th November 2017 Accepted 19th January 2018

DOI: $10.1039 / c 7 r a 12506 b$

rsc.li/rsc-advances

\section{Nanofiller-conjugated percolating conductive network modified polymerization reaction characteristics of aromatic thermosetting copolyester resin $\uparrow$}

\author{
Mete Bakir, (iD) a Jacob L. Meyer, (DD ad Andre Sutrisno, (D) c James Economy ${ }^{\text {bd }}$ \\ and Iwona Jasiuk iD *a
}

Deliberately controlled interfacial interactions between incorporated nanofiller particles and host polymer backbone chains constitute a critical element in the realm of polymer nanocomposites with tailorable multifunctional properties. We demonstrate the physicochemical effects induced by graphene nanoplatelets (GNP) of different sizes on the condensation polymerization reaction of aromatic thermosetting copolyester (ATSP) through the formation of electrically conductive percolating networks as enabled by interfacial interactions. Carboxylic acid and acetoxy-capped precursor oligomers of ATSP are solid-state mixed with chemically pristine GNP particles at various loading levels. Upon in situ endothermic condensation polymerization reaction, crosslinked backbone of the ATSP foam matrix is formed while the carbonaceous nanofillers are incorporated into the polymer network via covalent conjugation with functional end-groups of the oligomers. The controlled GNP size promotes different electrical percolation thresholds and ultimate electrical conductivities. Microstructural analysis demonstrates GNP distributions in the matrix as well as morphological modifications induced by the formation of conductive percolating GNP networks. Cure characteristics reveal the thermochemical changes prompted in the polymerization processes for GNP content above the requirement for percolation formation. Chemical spectroscopy of the ATSP nanocomposite morphology exhibits the formation of a robust interfacial coupling mechanism between the GNPs and ATSP backbone. The findings here may guide the developmental efforts of nanocomposites through better identifying roles of the morphology and content of nanofillers in polymerization processes.

\section{Introduction}

Technological advancements of structural materials currently count on the development of alternative lightweight materials possessing physical properties commensurate with contemporary systems while enabling cost-effective and industrially scalable high-throughput production solutions. ${ }^{\mathbf{1 - 4}}$ Polymer nanocomposites are combinations of nanofiller reinforcement particles and host polymer matrix, which uniquely embody multifunctional properties within low-density morphologies. ${ }^{5-7}$

${ }^{a}$ Department of Mechanical Science and Engineering, University of Illinois at Urbana-Champaign, Urbana, IL 61801, USA. E-mail: ijasiuk@illinois.edu; Tel: +1-217-333-92-59

${ }^{b}$ Department of Materials Science and Engineering, University of Illinois at UrbanaChampaign, Urbana, IL 61801, USA

${ }^{c} N M R / E P R$ Laboratory, School of Chemical Sciences, University of Illinois at UrbanaChampaign, Urbana, IL 61801, USA

${ }^{d}$ ATSP Innovations, Champaign, IL 61820, USA

$\dagger$ Electronic supplementary information (ESI) available. See DOI: 10.1039/c7ra12506b
Physical properties of the polymer nanocomposites can be adjusted by controlling the nanofiller loading at large. Yet, optimization of such properties involves many factors coming into effect including but not limited to processing conditions of the nanocomposites as well as morphology, dispersion quality, and chemical functionalization of the nanofillers. ${ }^{8-11}$ In this regard, recent efforts have sought to realize robust interfacial attachment schemes between edge-or-surface-functionalized nanofillers and backbone chains of various polymers. ${ }^{\mathbf{1 2}}$ Yet, current nanocomposite configurations are limited in terms of their ultimate physical properties due to the presence of only weak intermolecular coupling mechanisms. ${ }^{13}$

In particular, electrically conductive polymer nanocomposites have been a subject of extensive studies owing to their immense potential for broad spectrum of applications spanning batteries, membranes, and electromagnetic shielding. ${ }^{14-16}$ Within this framework, the formation of conductive networks of interconnected nanofillers - past the electrical percolation transition threshold - within intrinsically insulating polymer domains is imperative as far as the 
performances of the electrically conductive nanocomposites are concerned. ${ }^{17}$ The percolation threshold is recognized to vary with respect to morphology, size, and distribution of nanofillers as well as rheological characteristics of resins. ${ }^{18}$ On the other hand, it is well acknowledged that the increased nanofiller loading fractions, near or above the percolation transition, substantially deteriorate the structural integrity of the nanocomposites. ${ }^{19}$ Hence, a primary research thrust has been centered on efforts to realize minimal loading levels for percolation thresholds utilizing various types of nanofillers and polymer combinations prepared via different processing methods. ${ }^{20,21}$ Although, there are comprehensive studies reporting on the rheological properties of the nanocomposites as a result of incremented nanofiller contents, little is still known about the physicochemical effects of the nanofillers on characteristics of in situ polymerization processes. ${ }^{22}$

Aromatic thermosetting copolyester (ATSP) utilizes low cost, easily processable and crosslinkable oligomers to develop a high-performance polymer morphology (Scheme 1) ${ }^{23}$ The tailorable chemical structure of the backbone chain as well as convenient reconfigurability of the matrix into various form factors enable effectively controllable physical properties. ${ }^{24}$ Recently, ATSP nanocomposites have been introduced that facilitate improved distributions of nanofillers via a solid-state mixing route, which then result in substantially increased thermal and mechanical properties. ${ }^{5}$ In this work, we report on the physicochemical changes observed during in situ polycondensation reaction through formation of electrically conductive percolating GNP networks within the ATSP resin. ATSP nanocomposite foams are obtained through a polycondensation reaction between carboxylic acid- and acetoxy-<smiles>CC(=O)Oc1ccc(C(=O)O)cc1</smiles>

\section{b)}

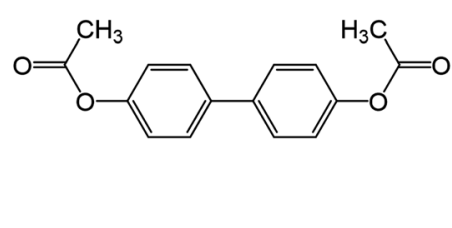

d)

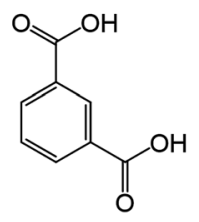

e)

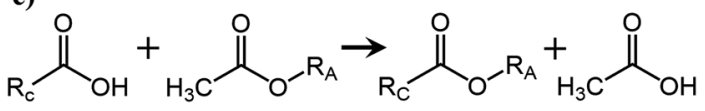

Scheme 1 Chemical structures of the monomers (a) 4-acetoxybenzoic acid (ABA), (b) biphenol diacetate (BDPA), (c) trimesic acid (TMA) and (d) isophthalic acid (IPA). (e) Chemical representation of the polycondensation reaction carried out between the acetoxy functionalized oligomer $\left(R_{\mathrm{A}}\right)$ (Fig. S1 $\uparrow$ ) and carboxylic acid functionalized oligomer $\left(R_{C}\right)$ (Fig. S2 $\dagger$ ) yielding a crosslinked polymer backbone and releasing acetic acid as a reaction by-product. capped constituent oligomers, which releases acetic acid as a reaction by-product to generate porous morphology. In the course of the in situ polymerization reaction, the GNPs covalently conjugate with the functionalized oligomers while the resin is advancing in molecular weight to develop the foam structures. The varied GNP size controls the electrical percolation transition thresholds and measured ultimate electrical conductivities. As well, microstructural analysis displays the morphological effects caused by the percolating networks of the GNPs. Cure characteristics reveal the influence of the nanofillers on the polymerization reaction. Chemical characterization of the nanocomposite backbone displays strong interfacial entanglement of the GNPs and ATSP matrix.

\section{Materials and experimental methods}

\subsection{Fabrication of aromatic thermosetting copolyester nanocomposites}

The constituent carboxylic acid and acetoxy functional group matching oligomers of the aromatic thermosetting copolyester are synthesized using biphenol diacetate (BPDA), 4-acetoxybenzoic acid (ABA), isophthalic acid (IPA), and trimesic acid (TMA) monomers (Sigma-Aldrich Co., USA) at particular molar feed ratios as further explained in earlier studies. ${ }^{23-25}$ The carboxylic acid $\left(\sim 1201 \mathrm{~g} \mathrm{~mol}^{-1}\right)$ and acetoxy-capped $\left(\sim 1203 \mathrm{~g} \mathrm{~mol}^{-1}\right.$ ) oligomers (Fig. S1, S2 and Table S1†) (premixed at $1: 1$ weight ratio) are combined in solid state with chemically pristine (non-functionalized) graphene nanoplatelet (GNP) particles of $1 \mu \mathrm{m}$ (thickness: 8-15 nm) (Cheap Tubes, Inc., USA), $5 \mu \mathrm{m}$ (thickness: 6-8 nm) (XG Sciences, Inc., USA), and $25 \mu \mathrm{m}$ (thickness: $6-8 \mathrm{~nm}$ ) (XG Sciences, Inc., USA) average flake diameters at $1,3,4,5,6,7,8$, and $10 \mathrm{wt} \%$ loading levels. ${ }^{5}$ The GNPs and ATSP precursor oligomers mixtures form powder combinations in solid state. The mixing process is performed via rigorous shaking until white oligomers powder turns to pitch dark, as also investigated under UV light. Such a color change indicates effective distribution of the nanofillers at macroscale in the oligomer powder bed. ${ }^{25}$ The ATSP nanocomposites are obtained via condensation polymerization (polycondensation) reaction between the constituent oligomers, which generates a crosslinked aromatic polyester backbone polymer network. ${ }^{25}$ The thermal polymerization process includes two dwell stages at $202{ }^{\circ} \mathrm{C}$ for 90 minutes and $270{ }^{\circ} \mathrm{C}$ for 150 minutes, respectively. The cycle has a final cure stage at $330{ }^{\circ} \mathrm{C}$ for 90 minutes. ${ }^{5}$ The nanocomposite foams are labeled as ATSP-GNP type as their naming convention.

\subsection{Physical characterization of aromatic thermosetting copolyester nanocomposites}

Solid-state nuclear magnetic resonance spectroscopy measurements are performed using ground nanocomposite specimens ( $\sim 50 \mathrm{mg}$ ) packed into NMR rotors with Unity Inova $300 \mathrm{MHz}$ NMR spectrometer (Varian Inc., USA). ${ }^{1} \mathrm{H}$ and ${ }^{13} \mathrm{C}$ spectra are acquired using direct pulse (DP) (pulse width (pw) $=2.5 \mu \mathrm{s}$, recycle time $(\mathrm{d} 1)=2 \mathrm{~s}$ ) and cross-polarized (CP) (pulse width 
$(\mathrm{pwH})=2 \mu \mathrm{s}$, recycle time $(\mathrm{d} 1)=2 \mathrm{~s}$, and contact time $(\mathrm{tHX})=4$ $\mathrm{ms}$ ) excitations, respectively. Specimens are spun at $10 \mathrm{kHz}$. Data analysis is performed using the MestreNova software. ${ }^{1} \mathrm{H}$ and ${ }^{13} \mathrm{C}$ NMR spectra are processed using $1 \mathrm{~Hz}$ and $25 \mathrm{~Hz}$ line broadenings, respectively.

Scanning Electron Microscopy (SEM) (S-4800, Hitachi, Japan) is operated in the high-resolution mode $(10 \mathrm{kV}$ voltage and $5 \mu \mathrm{A}$ current) to image microstructural features and distributions of the GNPs in the ATSP matrix.

Transmission electron microscopy is employed in brightfield mode $(200 \mathrm{kV}, 102 \mu \mathrm{A})$ to analyze the interfacial interactions of the GNPs with the ATSP chains (2010LaB 6 , JEOL, Japan). Fabricated nanocomposite specimens are ground using a laboratory grinder and the powder is settled in methanol solution for 10-15 min. Floating particles in the solution are collected using a pipette and passed through a copper grid, which is then dried at $80{ }^{\circ} \mathrm{C}$ for 1 hour.

Raman spectroscopy (Raman 11, Nanophoton, Japan) measurements are carried out using an excitation source of $633 \mathrm{~nm}$, a $20 \times$ objective lens, and a total acquisition time of 5 min.

Direct current (DC) electrical conductivity measurements are performed on foam morphology specimens using 4 point-probe method (6517 B, Keithley Instruments, USA). The specimens are in $5 \times 5 \times 12 \mathrm{~mm}^{3}$ (width $\times$ thickness $\times$ length). The DC electrical conductivity results are averaged over four samples per loading fraction, and standard deviations are given by error bars, accordingly. For the loading levels corresponding to percolation transitions, the nanocomposite specimens are labeled, in addition to the GNP size, with $<\varphi_{\mathrm{c}}, \sim \varphi_{\mathrm{c}},>\varphi_{\mathrm{c}}$ denoting below percolation, around percolation, and above percolation GNP contents, respectively.

Volumetric microstructural images are obtained on foam morphology specimens using a high-resolution X-ray microcomputed tomography (Micro-CT) (Xradia MicroXCT-400). The $3 \mathrm{D}$ objects are reconstructed utilizing total of 1441 images taken at every $0.25^{\circ}$ corresponding to sample rotation during the imaging process. The samples are in $10 \mathrm{~mm} \times 20 \mathrm{~mm}$ crosssection with $4 \mathrm{~mm}$ thickness.

Density is calculated as the ratio of measured weight to volume of the specimens. The density of neat fully dense ATSP is $1.27 \mathrm{Mg} \mathrm{m}^{-3}$.

The cure characteristics of the nanocomposite foams are investigated using a Differential Scanning Calorimetry (DSC) (DSC 2910, TA Instruments, USA). The tests are performed under an inert atmosphere of nitrogen. The cure cycle involves a temperature-ramped heating process with a $10{ }^{\circ} \mathrm{C} \mathrm{min}^{-1}$ heating rate.

\section{Results and discussion}

\subsection{Polymerization reaction and interfacial coupling mechanism}

First, we highlight the chemical principles of the polycondensation reaction carried out between the matching oligomer groups that form the crosslinked network of the ATSP matrix. The polymerization process involves the acetoxy and carboxylic acid-capped constituent oligomers conducting an esterification condensation reaction at sufficiently high temperatures exceeding $200{ }^{\circ} \mathrm{C}$ (for chemical structures of the oligomers see Fig. S1 and $\mathrm{S} 2 \dagger$ ). In the course of this reaction, ether oxygen groups of the acetoxy-capped oligomer exchange with the hydroxyl groups of the carboxylic acid-capped oligomer that produces the crosslinked aromatic backbone morphology while releasing acetic acid as a by-product, which consequently generates a porous morphology. ${ }^{25}$ Fig. 1 demonstrates solidstate Nuclear Magnetic Resonance (ssNMR) spectra of the individual carboxylic acid (C-group)- and acetoxy (A-group)capped oligomer groups, uncured combination of the oligomers ( $\mathrm{C}+\mathrm{A}$ mixed), and cured ATSP matrix (ATSP). In Fig. 1a, ${ }^{13} \mathrm{C}$ cross-polarization magic-angle spinning (CPMAS) spectra of the individual oligomers exhibit two main peak domains corresponding to aromatic main chains $(\mathrm{C}-\mathrm{C} / \mathrm{C}-\mathrm{H}$ bonds) and functional side chains $(\mathrm{C}-\mathrm{O} / \mathrm{C}=\mathrm{O}$ bonds). Measured chemical
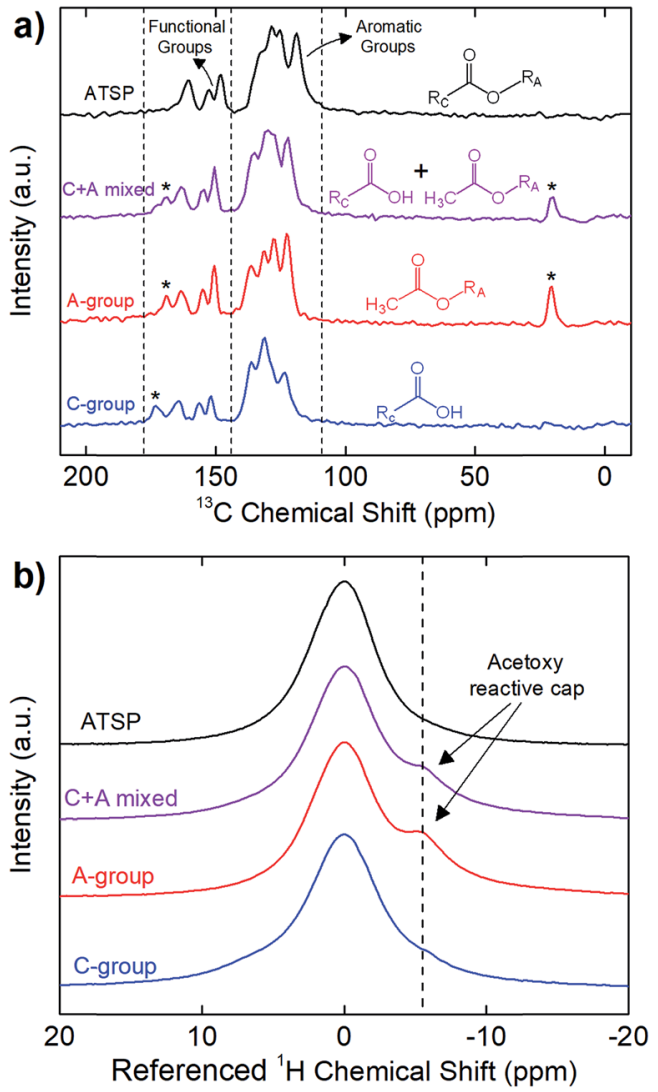

Fig. 1 Solid-state nuclear magnetic resonance (ssNMR) ${ }^{13} \mathrm{C}$ crosspolarization magic-angle spinning (CPMAS) (a) and ${ }^{1} \mathrm{H}$ direct pulse magic-angle spinning (DPMAS) (b) spectra of the carboxylic acid (Cgroup) and acetoxy (A-group) capped individual oligomers, uncured combination of the $C$ - and $A$-groups $(C+A$ mixed), and cured matrix (ATSP). Aromatic groups and functional groups represent $\mathrm{C}-\mathrm{C} / \mathrm{C}-\mathrm{H}$ and $\mathrm{C}-\mathrm{O} / \mathrm{C}=\mathrm{O}$ bonds participated in the oligomer sets as well as the cured matrix. $R_{\mathrm{C}}$ and $R_{\mathrm{A}}$ indicate aromatic chain configurations of the carboxylic acid and acetoxy group oligomers, respectively. Asterisks highlight the peaks associated with the reactive caps involving in the polymerization reaction. ${ }^{1} \mathrm{H}$ spectra referenced to zero to display the additional peak formations, corresponding chemical shift values are given in the text. 
shift positions of the C-group are $\delta=171.7,162.9,154.8,150.5$, 134.9, 129.9, and $122.1 \mathrm{ppm}$ and A-group are $\delta=168.7,162.8$, $154.8,150.5,136.2,131.2,127.3,122.1$, and $20.6 \mathrm{ppm}$. The solidstate uncured combination of the oligomers ( $\mathrm{C}+\mathrm{A}$ mixed $)$ displays similar characteristic peaks detected at $\delta=172.9$, 168.9, 162.9, 154.8, 150.5, 135.2, 129.9, 122.1, and $20.6 \mathrm{ppm}$. Following the polymerization reaction (performed outside of the NMR station), the cured ATSP morphology exhibits peaks at $\delta=160.5$, 152.6, 148.2, 132.9, 128.4, 125.2, 118.8 ppm. Hence, the two identified peak domains, emerging from the constituent oligomer groups, are effectively preserved within the ATSP morphology whereby a crosslinked aromatic backbone linked via oxygen bonds is formed. ${ }^{25}$ Regarding the chemical imprints of the reactive caps, the peaks observed at $\delta=171.7 \mathrm{ppm}$ in Cgroup, and $\delta=168.7$ and $20.6 \mathrm{ppm}$ in A-group (as indicated with asterisks in the figure) likewise transmit to the spectrum of the uncured mixture, yet disappear upon polymerization, and are absent from the ${ }^{13} \mathrm{C}$ spectrum of the cured ATSP matrix. It highlights that these peaks are associated with the reactive caps that participate in the reaction. In particular, the ether oxygen of the acetoxy cap (with the methyl $\left(-\mathrm{CH}_{3}\right)$ group displays at $\delta=$ $20.6 \mathrm{ppm}$ and the oxygen-bearing groups $(\mathrm{C}-\mathrm{O} / \mathrm{C}=\mathrm{O})$ display at $\delta=168.7 \mathrm{ppm}$ as observables) interacts with the hydroxyl group of the carboxylic acid cap (with the oxygen-bearing groups $(\mathrm{C}-\mathrm{O} /$ $\mathrm{C}=\mathrm{O}$ ) show a peak at $\delta=171.7 \mathrm{ppm}$ ) in the course of the polycondensation reaction which then releases acetic acid foaming as the by-product while the crosslinked morphology is formed. ${ }^{26}$ Besides, in Fig. $1 \mathrm{~b},{ }^{1} \mathrm{H}$ direct pulse magic-angle spinning (DPMAS) spectra show chemical shifts of the Cgroup at $\delta=7.4 \mathrm{ppm}, \mathrm{A}$-group at $\delta=7.4,1.7 \mathrm{ppm}, \mathrm{C}+\mathrm{A}$ mixed at $\delta=7.4,2.1 \mathrm{ppm}$, and ATSP at $\delta=5.0 \mathrm{ppm}$ (the shifts are referenced to zero in the figure for clarity). Particularly, the spectrum of the A-group displays a shoulder formation (at $\delta=$ $1.7 \mathrm{ppm}$ ) over the main peak, which represents the acetoxy based reactive cap of the oligomer. ${ }^{27}$ Note that although the acetoxy cap is detected in the ${ }^{1} \mathrm{H}$ spectrum of the uncured mixture, it is no longer observable after the esterification process. Hence, based on both ${ }^{1} \mathrm{H}$ and ${ }^{13} \mathrm{C}$ spectra, the formation of the crosslinked aromatic backbone is mainly controlled by the functional groups of the constituent oligomers reacting at elevated temperatures during the polymerization reaction.

Next, we demonstrate the in situ intermolecular attraction and interfacial coupling mechanism effective between the GNP nanoparticles and ATSP backbone chains, as shown in Fig. 2. During the thermal polymerization reaction between the constituent oligomers, the GNP particles interact with the reactive functional groups of the oligomers, and consecutively integrate with the crosslinked network upon curing. The underlying in situ interaction mechanism stems from intermolecular attraction forces between highly polar acetoxy and carboxylic acid caps of the oligomers and inherently oxygencontaining polar sites on the GNP particles. ${ }^{28}$ Within this scheme, molten oligomers at high temperatures blend together and wet the GNP particles through effective hydrodynamic forces emerging during the acetic acid by-product release. ${ }^{5}$ It then facilitates the polar force induced interfacial interaction between the GNPs and ATSP. Subsequently, the oligomers -
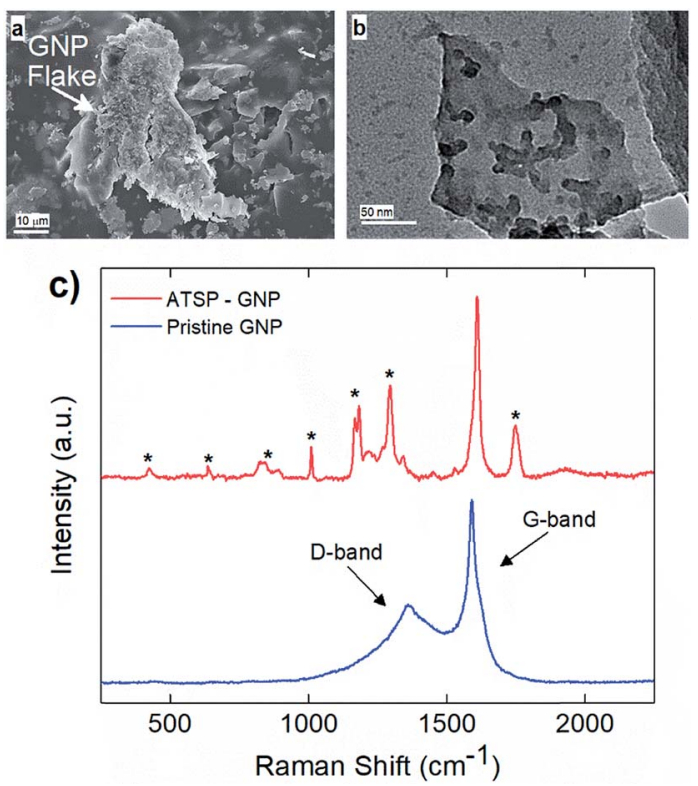

Fig. 2 A scanning electron microscopy image obtained on a single GNP flake (GNP $25 \mu \mathrm{m}$ ) thickly coated by ATSP matrix (a). A bright-field transmission electron microscopy image of a GNP flake (GNP $5 \mu \mathrm{m}$ ) decorated with interfacially tethered ATSP chain fragments (b). Raman spectra of pristine GNP flakes (GNP $5 \mu \mathrm{m}$ ) and GNPs within the ATSP matrix (c). Asterisks denote additional peaks formed upon interactions with the ATSP backbone chains.

containing acetoxy and carboxylic acid reactive groups - crosslink with the oxygen-bearing polar sites on the GNP particles upon the cure process which effectively tethers the GNPs to the crosslinked domain of the ATSP. Since the interfacial coupling occurs through oxygen bonds, it enables high physicochemical stability which effectively enhances the thermomechanical properties of the ATSP nanocomposites. ${ }^{25}$ Scanning electron microscopy (SEM) analysis reveals that the GNP particles are thickly coated by the matrix indicating effective surface wettability enabled through the in situ attraction mechanism (Fig. 2a). Hence, the GNP particles are not phobic to the molten oligomer groups during the polymerization process. More importantly, such an intrinsic interaction scheme indicates that the GNPs can also modify the polymerization reactions characteristics, which will be discussed later in the text. To highlight the interfacial coupling effect, transmission electron microscopy (TEM) image displays a GNP flake having ATSP chain fragments (darker domains) tethered on the surface that clearly indicates the molecular level extent of the coupling formation (Fig. 2b). In addition, Raman spectroscopy measurements, as shown in Fig. 2c, display the pristine GNP spectrum as compared to that of the GNP interacted with the ATSP matrix. The pristine GNP produces a conventional spectrum with two characteristic peaks at $1360 \mathrm{~cm}^{-1}$ and $1590 \mathrm{~cm}^{-1}$ which correspond to D (disordered hybridized structure with impurities) and $\mathrm{G}$ (ordered graphitic structure) bands, respectively. ${ }^{29}$ Upon the GNP particles interacting with the ATSP backbone chains, additional peak formations are observed, as denoted with asterisks. The peaks arise from the polymer domain for which 
the polymer chains are excited while strongly attached to the GNP structure (see Fig. S3† for Raman spectrum of the neat ATSP). Also note that the presence of the additional peaks disturbs the $\mathrm{D}$ band region, which indicates that the grafting takes place through a disordered area of the GNP flakes where the oxygen-containing sites are present. Hence, it is clearly evident that the GNP and ATSP matrix constitute a strong interfacial attachment. We note that the GNP particles remained intact in the ATSP matrix upon the polymerization reaction which apparently did not exfoliate, based on X-ray diffraction (XRD) spectroscopy demonstrated in an earlier work. ${ }^{5}$ Additionally, temperature-driven hydrodynamic motion within molten oligomer domain caused relocation of the acetic acid blowing agent bubbles enabling redistribution and rearrangement of the GNP particles prior to the curing that minimized nanoparticle aggregation in the nanocomposite matrix. ${ }^{5}$

\subsection{Electrical percolation characteristics}

Afterwards, we investigate the electrical percolation characteristics of the ATSP nanocomposites incorporated with the GNP particles of $1 \mu \mathrm{m}, 5 \mu \mathrm{m}$, and $25 \mu \mathrm{m}$ sizes. Direct Current (DC) electrical conductivity results of the ATSP nanocomposites obtained employing a four-point probe method are shown in Fig. 3. The neat ATSP (without addition of the GNPs) displays a characteristic non-conductive behavior with a DC conductivity of $4.04 \times 10^{-13} \pm 7.73 \times 10^{-14} \mathrm{~S} \mathrm{~m}^{-1}$ being in agreement with similar polymer systems. ${ }^{30}$ Upon selectively incremented nanofiller content, the electrical conductivity remains almost constant until the percolation threshold transitions of each GNP sizes. The percolation thresholds are observed to vary with respect to the GNP particle sizes. In particular, GNP $1 \mu \mathrm{m}$ forms the conductive percolating network around $5 \mathrm{wt} \%$ while GNP 5 $\mu \mathrm{m}$ and GNP $25 \mu \mathrm{m}$ obtain the interconnected domains near $4 \mathrm{wt} \%$ and $3 \mathrm{wt} \%$, respectively. The percolation thresholds are visually determined with respect to the S-shape characteristic

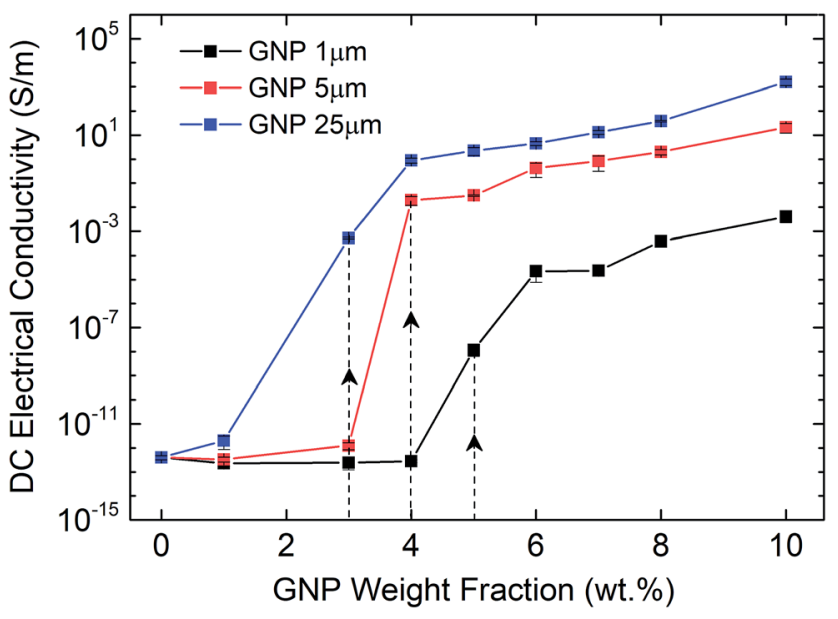

Fig. 3 Results of DC electrical conductivity measurements on the ATSP-GNP nanocomposites with GNPs of $1 \mu \mathrm{m}, 5 \mu \mathrm{m}$, and $25 \mu \mathrm{m}$. Standard deviations were represented with error bars. Dashed lines indicate assigned percolation thresholds for each GNP size. curve of the percolation transition. The observed percolation thresholds concur with the findings in the literature. ${ }^{31-33}$ The downshifts in the percolation thresholds with the larger GNPs occur due to the prompt development of the percolating networks which increases the probability of the formation of nanoparticle conductive paths in the matrix. ${ }^{34}$ The generalized connectedness percolation theory describes that the percolation threshold is independent of the aspect ratio for monodisperse disc-shaped platelet nanofillers. ${ }^{17,35}$ Yet, from an experimental point of view, difference in the average diameters of the GNPs (polydispersity) is expected for each nanocomposite, although they are formed through a single GNP size, considering the bulk manufacturing processes of the pristine GNPs as well as the processing conditions of the nanocomposites. Hence, the shifts in the percolation thresholds with respect to different GNP sizes are conceivable. In this regard, as polydispersity ratio (the variation of the GNP size for a certain given diameter) is increased, in this case through the presence of the larger GNP particles (assuming same thicknesses for all sizes), the percolation threshold decreases. ${ }^{\mathbf{1 7}}$ Above the percolation transitions, the nanocomposites acquire highly conductive states. As a matter of fact, we observe that the $10 \mathrm{wt} \%$ of GNP loading level displays electrical conductivities of $4.08 \times 10^{-3} \pm 1.81 \times 10^{-3} \mathrm{~S}$ $\mathrm{m}^{-1}$ for GNP $1 \mu \mathrm{m}, 21.2 \pm 9.08 \mathrm{~S} \mathrm{~m}^{-1}$ for GNP $5 \mu \mathrm{m}$, and $1.6 \times$ $10^{3} \pm 4.7810^{2} \mathrm{~S} \mathrm{~m}^{-1}$ for GNP $25 \mu \mathrm{m}$. A possible reason which gives rise to lower electrical conductivities with the smaller aspect ratio of the GNPs is the increased interfacial contact resistance through larger contact area. ${ }^{36}$ Another reason may be the different intrinsic electrical conductivities for each different GNP size induced through the presence of inherent functional groups or defect sites, yet conclusive remarks require further analysis. Based on the electrical conductivity results, we determine 3 percolation-based GNP contents for further physical analysis: below percolation $\left(<\varphi_{\mathrm{c}}\right)$, around percolation $\left(\sim \varphi_{\mathrm{c}}\right)$, and above percolation $\left(>\varphi_{\mathrm{c}}\right)$. For GNP $1 \mu \mathrm{m},\left\langle\varphi_{\mathrm{c}}, \sim \varphi_{\mathrm{c}},>\varphi_{\mathrm{c}}\right.$ correspond to $1 \mathrm{wt} \%, 5 \mathrm{wt} \%$, and $10 \mathrm{wt} \%$, respectively. For GNP $5 \mu \mathrm{m}$, $<\varphi_{\mathrm{c}}, \sim \varphi_{\mathrm{c}},>\varphi_{\mathrm{c}}$ correspond to $1 \mathrm{wt} \%, 4 \mathrm{wt} \%$ and $10 \mathrm{wt} \%$, respectively. For GNP $25 \mu \mathrm{m},<\varphi_{\mathrm{c}}, \sim \varphi_{\mathrm{c}},>\varphi_{\mathrm{c}}$ correspond to $1 \mathrm{wt} \%$, $3 \mathrm{wt} \%$, and $10 \mathrm{wt} \%$, respectively.

To further interrogate the percolation characteristics, we perform microstructural analysis via a Scanning Electron Microscope (SEM) on a set of specimens with GNP contents of below percolation $\left(<\varphi_{\mathrm{c}}\right)$, around percolation $\left(\sim \varphi_{\mathrm{c}}\right)$, and above percolation $\left(>\varphi_{\mathrm{c}}\right)$. As shown in Fig. $4 \mathrm{a}-\mathrm{c}$, GNP $1 \mu \mathrm{m}$ exhibits very random and wide distributions until the percolation threshold after which the GNP flakes are observed to effectively cover the surface of the matrix. In addition, in Fig. 4d-f, GNP $5 \mu \mathrm{m}$ specimens are observed to form some small clusters below the percolation point owing to the larger particle size. Upon increases in the content, the GNPs are embedded in the matrix. As well, above the percolation transition, the GNP particles are thickly coated with ATSP while dispersed over the matrix. In Fig. $4 \mathrm{~g}-\mathrm{i}$, GNP $25 \mu \mathrm{m}$ specimens display similar characteristics as GNP $5 \mu \mathrm{m}$, yet due to the larger particle size formations of cluster islands are clearly marked. The findings here indicate that the GNPs of the larger sizes ( $5 \mu \mathrm{m}$ and $25 \mu \mathrm{m}$ ) form cluster through percolation formation, significantly interact with the 

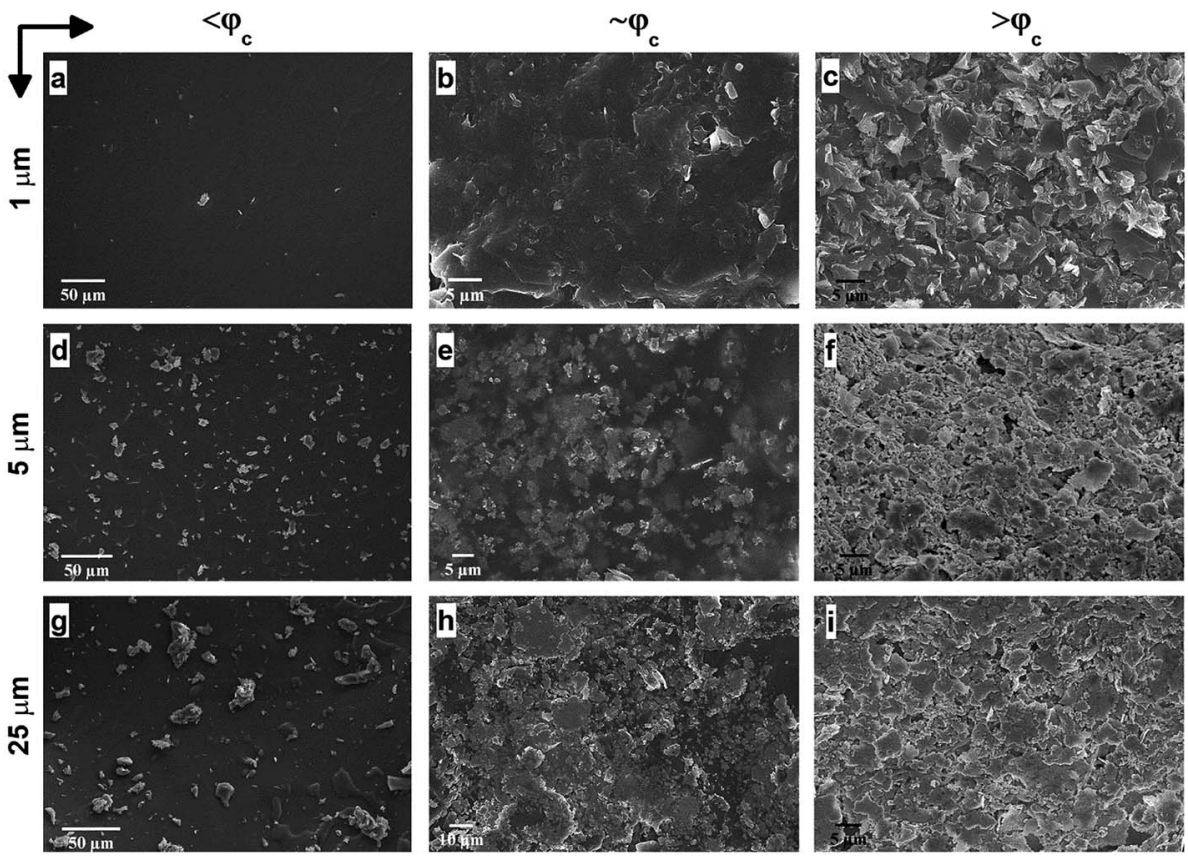

Fig. 4 Scanning electron microscopy (SEM) images of the ATSP-GNP nanocomposites highlighting differences in surface morphologies and nanoparticle distributions for the GNPs of $1 \mu \mathrm{m}(\mathrm{a}-\mathrm{c}), 5 \mu \mathrm{m}(\mathrm{d}-\mathrm{f})$ and $25 \mu \mathrm{m}(\mathrm{g}-\mathrm{i})$ below percolation $\left(<\varphi_{\mathrm{c}}\right)$, around percolation $\left(\sim \varphi_{\mathrm{c}}\right)$, and above percolation ( $>\varphi_{\mathrm{C}}$ ) thresholds. For GNP $1 \mu \mathrm{m},<\varphi_{\mathrm{C}}, \sim \varphi_{\mathrm{C}^{\prime}}>\varphi_{\mathrm{C}}$ correspond to $1 \mathrm{wt} \%, 5 \mathrm{wt} \%$, and $10 \mathrm{wt} \%$, respectively. For GNP $5 \mu \mathrm{m},<\varphi_{\mathrm{C}}, \sim \varphi_{\mathrm{C}},>\varphi_{\mathrm{C}}$ correspond to $1 \mathrm{wt} \%, 4 \mathrm{wt} \%$, and $10 \mathrm{wt} \%$, respectively. For GNP $25 \mu \mathrm{m},<\varphi_{\mathrm{c}}, \sim \varphi_{\mathrm{c}},>\varphi_{\mathrm{c}}$ correspond to $1 \mathrm{wt} \%, 3 \mathrm{wt} \%$, and $10 \mathrm{wt} \%$, respectively.

matrix, and effectively alter the topological features of the nanocomposite morphologies. Also, from a modeling perspective, the establishment of the percolating networks through the formation of clusters (defined as connectedness) for the larger GNPs significantly affects the percolation transition characteristics as well as the ultimate electrical conductivities. As such, the larger size of the clusters gives rise to the lower percolation transition in the non-conductive matrices, which is completely analogous to the polydispersity effect. ${ }^{37}$ We subsequently carry out a supplementary visual characterization on the nanocomposites having the corresponding GNP loading fractions to observe modifications in the volumetric morphologies. In Fig. S4, $\uparrow$ we show micro computed tomography (micro-CT) 3D reconstructed scanning images of the ATSP-GNP foam morphology nanocomposites. For GNP $1 \mu \mathrm{m}$, the intrinsic porous morphology does not change with respect to the formation of the percolating network. On the other hand, GNPs of $5 \mu \mathrm{m}$ and $25 \mu \mathrm{m}$ demonstrate notable changes in their morphologies obtaining nearly densified structural forms above the percolation transitions (see Table $\mathrm{S} 2 \dagger$ for calculated densities of the nanocomposites).

\subsection{Thermal cure characteristics and backbone chain modifications}

To investigate the chemical basis that causes the structural modifications with the increased GNP contents, we analyze the thermal characteristics of the endothermic condensation polymerization reaction at the percolation-determined loading levels. The combined mixtures of the precursor oligomers with the GNPs are subjected to a temperature-ramp differential scanning calorimetry (DSC) cycle at a $10{ }^{\circ} \mathrm{C} \mathrm{min}^{-1}$ constant heating rate. The obtained thermal profiles of the GNP combined mixtures are then compared to that of the neat mixture of the constituent oligomers. ${ }^{25}$ In Fig. 5, we compare only the above-percolation GNP content to the parent material to highlight the thermochemical changes that are effective for

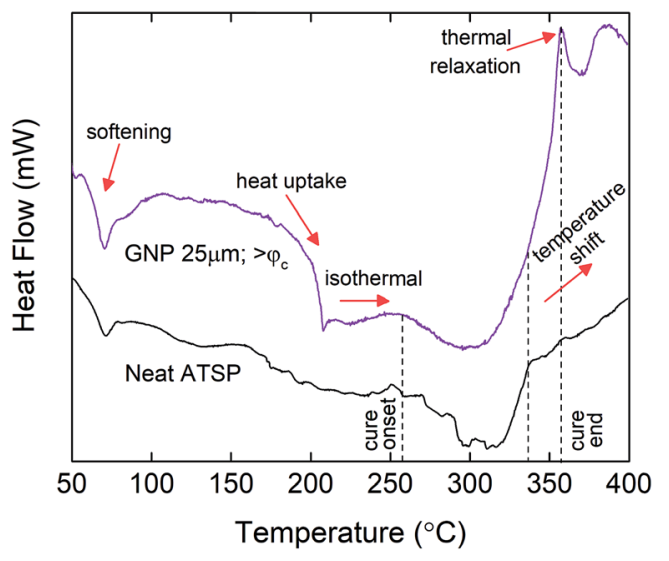

Fig. 5 Differential scanning calorimetry (DSC) thermal characteristics of the condensation polymerization reaction for the neat mixture of the precursor oligomers and combined mixture of the oligomers with GNP $25 \mu \mathrm{m}$ having $>\varphi_{\mathrm{c}}$ loading level $(10 \mathrm{wt} \%)$. DSC curves are arbitrarily shifted to highlight differences. For comprehensive results of all the GNP sizes at given percolation-determined loading levels, please refer to ESI (Fig. S4 $\dagger$ ). Tests are performed under an inert atmosphere of nitrogen. The heating rate is $10^{\circ} \mathrm{C} \mathrm{min}^{-1}$. 
other GNP sizes. For comprehensive results of all the GNP sizes at given percolation-determined loading levels, please refer to ESI (Fig. S5-S7†). As shown in Fig. 5, at the beginning of the heating processes, the heat flux curve of the GNP combined mixture likewise reveals a dimple around $70{ }^{\circ} \mathrm{C}$ denoting structural softening of the constituent oligomers, which is similarly observed in the base formulation. ${ }^{25}$ Afterwards, especially for GNP $1 \mu \mathrm{m}$, the thermal profile maintains similarly endothermic patterns akin to the neat mixture during which melting and polycondensation reaction stages accordingly take place (Fig. S5 $\dagger$ ). However, both GNP $5 \mu \mathrm{m}$ and GNP $25 \mu \mathrm{m}$ reveal significant heat uptakes into the molten oligomer domain, which are particularly observed for the above-percolation loading levels $\left(>\varphi_{\mathrm{c}}\right)$ (Fig. S6 and S7 $\dagger$ ). Such abrupt behaviors are related to acetic acid discharge-driven bubble growth during the condensation reaction that is greatly affected by the presence of the carbon fillers. ${ }^{38}$ Following this stage, the cure process initiates (denoted as cure onset) around at $260{ }^{\circ} \mathrm{C}$ for the parent form in which the thermal curve displays a genuine endothermic cure region. ${ }^{25}$ For GNP $1 \mu \mathrm{m}$, only the $>\varphi_{\mathrm{c}}$ loading level exhibits a distinctly broader cure region than the neat oligomers for which the completion of the cure process extends beyond $350{ }^{\circ} \mathrm{C}$ (Fig. S5†). In addition, upon completion of the cure process, a thermal relaxation peak, a small hump (denoted as cure end, indicated with an arrow) is observed. Likewise, for both GNP $5 \mu \mathrm{m}$ and GNP $25 \mu \mathrm{m}$, similar broadening of the cure domains as well as the peak formations are observed at abovepercolation loading levels. The broadening of the cure region is likely to occur due to increased melt viscosity of the oligomers caused by the presence of the nanofillers that required more energy input to carry out the crosslinking process, which could subsequently stimulate an early gelation of the crosslinked matrix..$^{39}$ Also, the reactive functional groups of the oligomers graft onto carbon nanofillers that can alter viscous characteristics of the molten domain during curing. ${ }^{40}$ The thermal relaxation peaks form due to devitrification of the matrix in the thermal process during which the glass transition temperature increased above the cure temperature. ${ }^{41}$

In addition, in Fig. 6, we demonstrate solid-state Nuclear Magnetic Resonance (ssNMR) spectroscopy measurements performed on above-percolation content of GNP $25 \mu \mathrm{m}$ in comparison to the parent material to identify chemical modifications in the backbone chain configuration as well as manifesting the interfacial attachment scheme in the nanofiller incorporated matrix. For comprehensive results of all the GNP sizes at given percolation-determined loading levels, refer to ESI (Fig. S8-S10†). ${ }^{13} \mathrm{C}$ cross-polarization magic-angle spinning (CPMAS) spectrum of the parent material (neat ATSP) display the two characteristic peak domains: the aromatic groups (C-C and $\mathrm{C}-\mathrm{H})$ and the functional side groups $(\mathrm{C}-\mathrm{O}$ and $\mathrm{C}=\mathrm{O})$, with a line width of $\sim 860 \mathrm{~Hz}$ over the highest intensity peak ( $\sim 130 \mathrm{ppm})$ $\left({ }^{13} \mathrm{C}\right.$ NMR spectra are processed using $25 \mathrm{~Hz}$ line broadening). ${ }^{1} \mathrm{H}$ direct pulse magic-angle spinning (DPMAS) NMR spectrum of the neat ATSP shows a single characteristic having a line width of $\sim 1640 \mathrm{~Hz}\left({ }^{1} \mathrm{H}\right.$ NMR spectra are processed using $1 \mathrm{~Hz}$ line broadening). Based on these NMR analyses, we observe that both ${ }^{1} \mathrm{H}$ and ${ }^{13} \mathrm{C}$ spectra of the nanocomposites with GNPs $5 \mu \mathrm{m}$
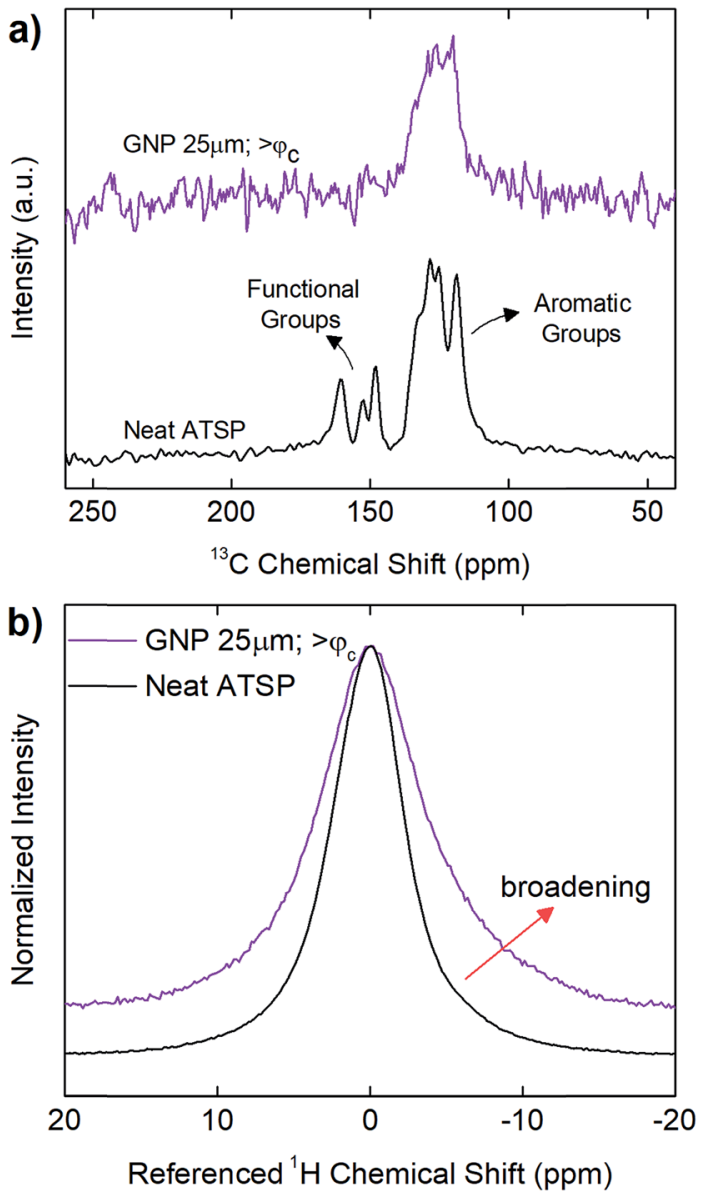

Fig. 6 Solid-state nuclear magnetic resonance (ssNMR) ${ }^{13} \mathrm{C}$ crosspolarization magic-angle spinning (CPMAS) (a) and ${ }^{1} \mathrm{H}$ direct pulse magic-angle spinning (DPMAS) (b) spectra of the neat ATSP morphology with respect to ATSP nanocomposites with GNP $25 \mu \mathrm{m}$ having $>\varphi_{\mathrm{c}}$ loading level (10 wt\%). ${ }^{1} \mathrm{H}$ and ${ }^{13} \mathrm{C}$ NMR spectra are processed using $1 \mathrm{~Hz}$ and $25 \mathrm{~Hz}$ line broadenings, respectively. The figures were given in normalized intensity axes.

(Fig. S9†) and $25 \mu \mathrm{m}$ (Fig. 6 and S10†) reveal clearly broadened characteristic peaks with respect to the neat parent matrix. For ${ }^{1} \mathrm{H}$, GNP $5 \mu \mathrm{m}$ demonstrates a line width of $2257 \mathrm{~Hz}$ at percolation content in comparison to $1640 \mathrm{~Hz}$ of the neat matrix. Similarly, for ${ }^{13} \mathrm{C}$, GNP $5 \mu \mathrm{m}$ displays a line width of $1528 \mathrm{~Hz}$ at percolation content with respect to $860 \mathrm{~Hz}$ of the neat ATSP. Whereas GNP $1 \mu \mathrm{m}$ display characteristic peaks similar to the base ATSP, yet decreased line widths with respect to increased GNP content may indicate only moderate interactions with the matrix (Fig. S8 $\dagger$ ). For GNPs $5 \mu \mathrm{m}$ and $25 \mu \mathrm{m}$, such peak broadening is formed due to strong interfacial coupling between the GNP particles and ATSP backbone chain, which then modifies the structural relaxation behavior of the nanocomposite matrix. ${ }^{42,43}$ In particular, the attachment scheme incurs an electron mobility difference due to individually dissimilar relaxation times of highly conductive GNP particles and highly dielectric ATSP matrix. More importantly, we do not observe any characteristic peaks in ${ }^{13} \mathrm{C}$ spectra coming from the matrix of GNP $5 \mu \mathrm{m}$ at above-percolation loading level (Fig. S9†). As well, ${ }^{13} \mathrm{C}$ spectrum of the GNP $25 \mu \mathrm{m}$ for $>\varphi_{\mathrm{c}}$ form a single slightly 
broader peak associated with only the aromatic group of the backbone configuration (Fig. 6 and S10†). Hence, the presence of larger GNP particles alter polymerization process at a significant extent, as also justified with the above-mentioned results.

Based on the characterization results presented here, we understand that due to the interfacial attachment mechanism coming into effect during the polymerization reaction, a fraction of the functional groups of the oligomer structures are reacted with the GNP particles (especially $5 \mu \mathrm{m}$ and $25 \mu \mathrm{m}$ ), which consequently reduces the fraction of the functional groups that participate in the ordinary crosslinking polymerization process. Indeed, the GNP-coupled configurations of the oligomers prompt an early gelation point during the polymerization reaction without necessitating completion of the crosslinking process. As a consequence of the gelation, the DSC profiles of $5 \mu \mathrm{m}$ and $25 \mu \mathrm{m}$ with loadings near and above their percolation thresholds display nearly flat regions following the sudden heat uptakes until cure onset. Under such a circumstance, the modified polymerization process does not solely perform the esterification reaction, which conjugates the two oligomer groups releasing acetic acid by product. This reduces the foaming agent released during the cure process, rendering higher density morphologies as observed in GNP $5 \mu \mathrm{m}$ and 25 $\mu \mathrm{m}$ above percolation transitions. In this context, the temperature-wise broadening characteristic observed in the cure regions indicates that the advancing crosslinking resin requires more heat input to activate unreacted bonds to complete the cure process. Due to the effect of such an interfacial entanglement mechanism, glass transition characteristics of similar polymer nanocomposites are demonstrated to be modified displaying significant temperature shifts. ${ }^{13}$ Hence, in this case, the glass transition temperature $\left(\sim 191{ }^{\circ} \mathrm{C}\right)$ may positively shift to a higher temperature in the cure region such that the polymer chains display the glass transition relaxation while the isothermal cure process is still in progress. ${ }^{25}$ Therefore, the peak formation at cure end point takes place due to devitrification (phase transformation from glassy state to rubbery state upon the glass transition) of the polymer network. Note that the vitrification induces slower cure rates wherein the cure reaction becomes more diffusion controlled rather than kinetic controlled, which also correlates to the cure region temperature broadening. ${ }^{44}$ Characterization of rheological properties of the nanofiller incorporated ATSP resin during the in situ polymerization reaction will be the subject of a follow-up study.

\section{Conclusions}

We present the physicochemical characteristic effects influenced by the GNPs of different sizes on the polycondensation reaction of the ATSP resin via the formation of electrically conductive percolating network. During the polymerization process, carboxylic acid and acetoxy-capped precursor oligomers, combined with the GNPs, form the crosslinked aromatic backbone of the ATSP matrix while the nanofillers interact with the reactive caps of the oligomers and combine with the crosslinked polymer network. The varied GNP size causes different electrical percolation thresholds and ultimate electrical conductivities. Microstructural characterization displays the GNP distributions in the matrix as well as morphological modifications formed through the conductive percolating networks. Cure characteristics reveal that the thermochemical changes occurred on the polymerization processes. SsNMR spectroscopies on the ATSP nanocomposite morphology exhibit the formation of a robust interfacial coupling mechanism between the GNPs and ATSP backbone.

\section{Conflicts of interest}

There are no conflicts of interest to declare.

\section{Acknowledgements}

We acknowledge characterization instruments provided at Frederick Seitz Materials Research Laboratory, Mechanical Testing Instructional Laboratory, Nuclear Magnetic Resonance/ Electron Paramagnetic Resonance Laboratory, and Beckman Institute for Advanced Science and Technology at the University of Illinois at Urbana-Champaign, and thank research staff members for their help with the experiments. We sincerely thank Prof. Alexey Bezryadin and Prof. Eduard Ilin for the help with the electrical conductivity measurements. We gratefully acknowledge funding from the National Science Foundation (NSF) I/UCRC grant (IIP-1362146). The findings, conclusions, and recommendations expressed in this manuscript are those of the authors and do not necessarily reflect the views of the NSF.

\section{References}

1 J. C. Williams and E. A. Starke, Acta Mater., 2003, 51, 57755799.

2 T. M. Pollock, Science, 2010, 328, 986-987.

3 J. Hirsch, Mater. Trans., 2011, 52, 818-824.

4 M. Telford, Mater. Today, 2004, 7, 36-43.

5 M. Bakir, J. L. Meyer, J. Economy and I. Jasiuk, Polymer, 2017, 123, 311-320.

6 P. Podsiadlo, A. K. Kaushik, E. M. Arruda, A. M. Waas, B. S. Shim, J. Xu, H. Nandivada, B. G. Pumplin, J. Lahann,

A. Ramamoorthy and N. A. Kotov, Science, 2007, 318, 80-83.

7 J. Jordan, K. I. Jacob, R. Tannenbaum, M. A. Sharaf and I. Jasiuk, Mater. Sci. Eng., R, 2005, 393, 1-11.

8 M. Moniruzzaman and K. I. Winey, Macromolecules, 2006, 39, 5194-5205.

9 V. Yudin, J. U. Otaigbe, V. Svetlichnyi, E. Korytkova, O. Almjasheva and V. Gusarov, eXPRESS Polym. Lett., 2008, 2, 485-493.

10 J. Liang, Y. Huang, L. Zhang, Y. Wang, Y. Ma, T. Guo and Y. Chen, Adv. Funct. Mater., 2009, 19, 2297-2302.

11 H. Kim, A. A. Abdala and C. W. Macosko, Macromolecules, 2010, 43, 6515-6530.

12 J. R. Potts, S. H. Lee, T. M. Alam, J. An, M. D. Stoller, R. D. Piner and R. S. Ruoff, Carbon, 2011, 49, 2615-2623. 
13 K.-H. Liao, S. Aoyama, A. A. Abdala and C. Macosko, Macromolecules, 2014, 47, 8311-8319.

14 H. Wu, G. Yu, L. Pan, N. Liu, M. T. McDowell, Z. Bao and Y. Cui, Nat. Commun., 2013, 4, 1943.

15 G. Shi, M. Rouabhia, Z. Wang, L. H. Dao and Z. Zhang, Biomaterials, 2004, 25, 2477-2488.

16 P. Karimi, M. Ostoja-Starzewski and I. Jasiuk, J. Appl. Phys., 2016, 120, 145103.

17 S. Kale, F. A. Sabet, I. Jasiuk and M. Ostoja-Starzewski, J. Appl. Phys., 2015, 118, 154306.

18 W. Zheng, S.-C. Wong and H.-J. Sue, Polymer, 2002, 43, 67676773.

19 A. Shalwan and B. Yousif, Mater. Des., 2014, 59, 264-273.

20 W. Bauhofer and J. Z. Kovacs, Compos. Sci. Technol., 2009, 69, 1486-1498.

21 Z. Spitalsky, D. Tasis, K. Papagelis and C. Galiotis, Prog. Polym. Sci., 2010, 35, 357-401.

22 P. Pötschke, T. Fornes and D. Paul, Polymer, 2002, 43, 32473255.

23 D. Frich, K. Goranov, L. Schneggenburger and J. Economy, Macromolecules, 1996, 29, 7734-7739.

24 B. Vaezian, J. L. Meyer and J. Economy, Polym. Adv. Technol., 2016, 27, 1006-1013.

25 M. Bakir, J. L. Meyer, J. Economy and I. Jasiuk, Macromolecules, 2016, 49, 6489-6496.

26 P. A. Couperus, A. D. H. Clague and J. P. C. M. van Dongen, Org. Magn. Reson., 1978, 11, 590-597.

27 W. Bubb, C. Fallick and S. Sternhell, Magn. Reson. Chem., 1977, 9, 167-174.

28 F. Wang, L. T. Drzal, Y. Qin and Z. Huang, J. Mater. Sci., 2015, 50, 1082-1093.
29 J. Xiang and L. T. Drzal, Polymer, 2012, 53, 4202-4210.

30 J. A. King, F. A. Morrison, J. M. Keith, M. G. Miller, R. C. Smith, M. Cruz, A. M. Neuhalfen and R. L. Barton, J. Appl. Polym. Sci., 2006, 101, 2680-2688.

31 J. B. Bai and A. Allaoui, Composites, Part A, 2003, 34, 689-694. 32 J. T. Choi, D. H. Kim, K. S. Ryu, H.-i. Lee, H. M. Jeong, C. M. Shin, J. H. Kim and B. K. Kim, Macromol. Res., 2011, 19, 809-814.

33 S. Kale, F. A. Sabet, I. Jasiuk and M. Ostoja-Starzewski, J. Appl. Phys., 2016, 120, 045105.

34 A. Aharony and D. Stauffer, Introduction to percolation theory, Taylor \& Francis, 2003.

35 R. H. J. Otten and P. v. d. Schoot, J. Chem. Phys., 2011, 134, 094902.

36 K. Nagata, H. Iwabuki and H. Nigo, Compos. Interfaces, 1998, 6, 483-495.

37 A. V. Kyrylyuk and P. van der Schoot, Proc. Natl. Acad. Sci. U. S. A., 2008, 105, 8221-8226.

38 P. Pötschke, T. D. Fornes and D. R. Paul, Polymer, 2002, 43, 3247-3255.

39 C.-Y. M. Tung and P. J. Dynes, J. Appl. Polym. Sci., 1982, 27, 569-574.

40 S. Li, M. Xiao, D. Wei, H. Xiao, F. Hu and A. Zheng, Polymer, 2009, 50, 6121-6128.

41 I. Fraga, S. Montserrat and J. M. Hutchinson, Macromol. Chem. Phys., 2008, 209, 2003-2011.

42 J. Chen, H. Liu, W. A. Weimer, M. D. Halls, D. H. Waldeck and G. C. Walker, J. Am. Chem. Soc., 2002, 124, 9034-9035.

43 A. D. Bain, D. R. Eaton, A. E. Hamielec, M. Mlekuz and B. G. Sayer, Macromolecules, 1989, 22, 3561-3564.

44 M. Kamal and S. Sourour, Polym. Eng. Sci., 1973, 13, 59-64. 